\title{
INVARIANT PROPERTIES OF THE GENERALIZED CANONICAL MAPPINGS
}

\author{
STANISAW JANECZKO \\ Institute of Mathematics, Warsaw University of Technology \\ Pl. Politechniki 1, 00-661 Warsaw, Poland \\ E-mail: janeczko@alpha.im.pw.edu.pl
}

Bogdan Ziemian in memoriam

\begin{abstract}
One of the fundamental objectives of the theory of symplectic singularities is to study the symplectic invariants appearing in various geometrical contexts. In the paper we generalize the symplectic cohomological invariant to the class of generalized canonical mappings. We analyze the global structure of Lagrangian Grassmannian in the product symplectic space and describe the local properties of generic symplectic relations.
\end{abstract}

1. Introduction. Let $\Omega$ be a smooth compact convex region in $\mathbb{R}^{\ltimes}$. Let $X$ be the boundary of $\Omega$ and $T^{*} X$ the cotangent bundle of $X$ (cf. [3, 11]). The symplectic billiard map $B: T^{*} X \rightarrow T^{*} X$ is defined on the set $U=\left\{(x, \xi) \in T^{*} X:|\xi|<1\right\}$ and as a Lagrangian submanifold, the graph of $B$, graph $B \subset\left(T^{*} X \times T^{*} X, \pi_{2}^{*} \theta_{X}-\pi_{1}^{*} \theta_{X}\right)$, is generated by the function

$$
\widetilde{H}: X \times X \rightarrow \mathbb{R}, \quad \widetilde{\mathbb{H}}\left(\curvearrowleft, \curvearrowleft^{\prime}\right)=\left|\curvearrowleft^{\prime}-\curvearrowleft\right|,
$$

where $\theta_{X}$ is the Liouville one-form on $T^{*} X$ and $\pi_{i}$ are the canonical projections. By the cotangent bundle projection $\pi_{X \times X}: T^{*} X \times T^{*} X \rightarrow X \times X$ and the smooth map $\rho: T^{*} X \rightarrow T^{*} X \times T^{*} X, \rho(x, \xi)=\left(x,-\xi, x^{\prime}, \xi^{\prime}\right)$, where $B(x, \xi)=\left(x^{\prime}, \xi^{\prime}\right)$, we get the function $H: T^{*} X \rightarrow R, H=\rho^{*} \pi_{X \times X}^{*} \widetilde{H}$ (normalized on $\bar{U}$ ) such that

$$
B^{*} \theta_{X}-\theta_{X}=d H \text {. }
$$

Consequently, if $\sigma=\left\{\left(x_{0}, \xi_{0}\right),\left(x_{1}, \xi_{1}\right), \ldots,\left(x_{k-1}, \xi_{k-1}\right)\right\}$ is a periodic orbit of $B$ then

$$
L(\sigma)=\sum_{j=0}^{k-1} H\left(x_{j}, \xi_{j}\right)
$$

1991 Mathematics Subject Classification: Primary 46C20. Secondary 32G81.

Research of the author supported by KBN grant No. 965 P03 9610.

The paper is in final form and no version of it will be published elsewhere. 
is a symplectic invariant. It is the length

$$
\left|x_{1}-x_{0}\right|+\ldots+\left|x_{0}-x_{k-1}\right|
$$

of the closed geodesic of the billiard map, and defines, for all closed orbits, the length spectrum of $\Omega$ (cf. [11]). If $\Omega$ is no longer convex, then the corresponding billiard map is not univalued and should be replaced by an appropriate symplectic relation, i.e. Lagrangian submanifold in $\left(T^{*} X \times T^{*} X, \pi_{2}^{*} \theta_{X}-\pi_{1}^{*} \theta_{X}\right)$, which is not the graph of any canonical mapping (see Figure 1 below). Symplectic relations (or symplectic correspondences $[4,7,2]$ ) play an important role in symplectic techniques in physics, geometric diffraction theory and singularity theory (cf. $[4,5,9,1])$.

In this paper, we show that the symplectic invariant $L(\sigma)$ may be generalized to the symplectic relation and for the special classes of them it is represented by the critical values of the corresponding generating function. Now there is a natural question concerning singularities of symplectic relations and their position with respect to the product structure. Following the singularity theory methods (cf. [1, 10]) and using the group of symplectomorphisms preserving the product structure as an equivalence group the generic properties of symplectic relations are investigated. At first, in Section 3, we study the canonical stratification of Lagrangian Grassmannian in the product symplectic space. Then, in Section 4, using the Lagrangian "Gauss map" on symplectic relations we describe the generic properties of them and obtain some prenormal forms in appropriate $k$-vertical points.

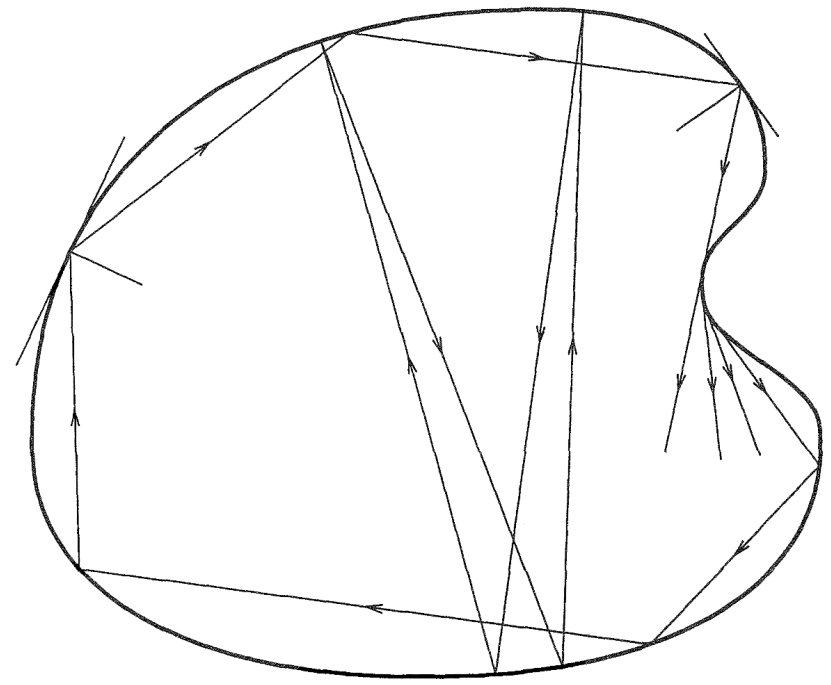

Figure 1

2. A cohomological invariant of symplectic relations. Let $\left(M_{1}, \omega_{1}\right),\left(M_{2}, \omega_{2}\right)$ be two symplectic manifolds. We consider the product symplectic manifold

$$
\mathcal{M}=\left(M_{1} \times M_{2}, \pi_{2}^{*} \omega_{2}-\pi_{1}^{*} \omega_{1}\right)
$$


where $\pi_{i}: M_{1} \times M_{2} \rightarrow M_{i}$ are canonical projections. Any Lagrangian submanifold $L$ of $\mathcal{M}$ will be called a symplectic relation between $M_{1}$ and $M_{2}$ (cf. $[17,18]$ ). We will assume $L$ is compact (with boundary and simply connected) and instead of $\left(M_{1}, \omega_{1}\right)$, $\left(M_{2}, \omega_{2}\right)$ we have two copies of the same symplectic manifold $(M, \omega)$ (we assume $M$ is a simply connected manifold). For any choice of $\alpha$ such that $\omega=d \alpha$ the form $\pi_{2}^{\star} \alpha-\left.\pi_{1}^{\star} \alpha\right|_{L}$ is exact. Let $H$ be a smooth function on $L$ such that

$$
\pi_{2}^{\star} \alpha-\left.\pi_{1}^{\star} \alpha\right|_{L}=d H .
$$

$H$ is unique up to an additive constant and we have to fix this constant in further considerations. We fix it assuming

$$
\int_{L} H\left(\left.\pi_{1}\right|_{L}\right)^{\star} \omega^{n}=0
$$

If $\alpha_{1}$ is another one-form for which $d \alpha_{1}=\omega$, then there exists a smooth function $G$ such that $\alpha_{1}-\alpha=d G$ ( $M$ has a boundary or is not compact). Now for a new underlying one-form $\alpha_{1}$ the Lagrangian submanifold $L$ has another generating function $H_{1}$

$$
\pi_{2}^{\star} \alpha_{1}-\left.\pi_{1}^{\star} \alpha_{1}\right|_{L}=d H_{1} .
$$

The relation between $H_{1}$ and $H$ is given by the formula

$$
H_{1}=H+\left.\left(\pi_{2}^{\star} G-\pi_{1}^{\star} G\right)\right|_{L}
$$

since we normalized $H_{1}$ and

$$
\int_{L} \pi_{2}^{\star} G\left(\left.\pi_{1}\right|_{L}\right)^{\star} \omega^{n}=\int_{L} \pi_{1}^{\star} G\left(\left.\pi_{1}\right|_{L}\right)^{\star} \omega^{n} .
$$

Let $\sigma=\left\{\left(x_{0}, x_{1}\right) \in L,\left(x_{1}, x_{2}\right) \in L, \ldots,\left(x_{k-1}, x_{0}\right) \in L\right\}$ be a periodic orbit of $L$. With $\sigma$ we will associate the number

$$
N(\sigma)=\sum_{i=0}^{k-2} H\left(x_{i}, x_{i+1}\right)+H\left(x_{k-1}, x_{0}\right) .
$$

Now we have

Proposition 2.1. The number $N(\sigma)$ is an invariant with respect to the action of the group of symplectomorphisms on $(M, \omega)$.

Proof. We have the following cyclic relation from formula (2):

$$
H_{1}\left(x_{i}, x_{i+1}\right)=H\left(x_{i}, x_{i+1}\right)+G\left(x_{i+1}\right)-G\left(x_{i}\right),
$$

so we obtain the invariance property for the number $N(\sigma)$,

$$
\begin{aligned}
\sum_{i=0}^{k-2} H_{1}\left(x_{i}, x_{i+1}\right)+H_{1}\left(x_{k-1}, x_{0}\right) & \\
=\sum_{i=0}^{k-2}\left(H\left(x_{i}, x_{i+1}\right)+G\left(x_{i+1}\right)-G\left(x_{i}\right)\right)+ & H\left(x_{k-1}, x_{0}\right)+G\left(x_{0}\right)-G\left(x_{k-1}\right) \\
& =\sum_{i=0}^{k-2} H\left(x_{i}, x_{i+1}\right)+H\left(x_{k-1}, x_{0}\right) .
\end{aligned}
$$


Remark 2.1.

A. If $L=\operatorname{graph} B$, where $B:(M, \omega) \rightarrow(M, \omega)$ is a symplectomorphism, then the set of numbers $\{N(\sigma)\}$ corresponding to the set of all periodic orbits of $B$ is called the spectrum of $B$. It is extensively studied in the case if $B$ is a billiard mapping associated with a convex region in $\mathbb{R}^{\ltimes}$ (cf. [3]). In the case of $L=\operatorname{graph} B$ formula (2) reduces to

$$
H_{1}=H+B^{\star} G-G \text {. }
$$

B. Now we assume that $x_{0}$ is a fixed point of $B$, i.e. $B\left(x_{0}\right)=x_{0}$. If $i:\left(S, s_{0}\right) \rightarrow\left(M, x_{0}\right)$ is an immersion germ of a smooth submanifold $S$ such that $\left.B^{(n)}\right|_{S}=\operatorname{id}_{S}$ for some $n \in N$, then the function

$$
S \ni s \mapsto H(s)+H(B(s))+\ldots+H\left(B^{(n-1)}(s)\right)
$$

is a symplectic invariant of the $\operatorname{star}\left\{\left(s, s_{1}, \ldots, s_{n-1}\right), x_{0}\right\}, S_{i}=B^{(i)}(S), i=1, \ldots, n-1$. Other invariants of Lagrangian stars were studied in $([6,15])$. If $S$ is a Lagrangian submanifold, then for 3-Lagrangian stars $\left\{\left(S, S_{1}, S_{2}\right), x_{0}\right\}$ which are pairwise transversal at $x_{0}$ the signature $\tau\left(S, S_{1}, S_{2}\right)$ of the quadratic form

$$
\omega\left(x_{1}, x_{2}\right)+\omega\left(x_{2}, x_{3}\right)+\omega\left(x_{3}, x_{1}\right)
$$

defined on the tangent (at $x_{0}$ ) Lagrangian star $T_{x_{0}} S \oplus T_{x_{0}} S_{1} \oplus T_{x_{0}} S_{2}$ is the symplectic invariant. This is the symplectic invariant for any Lagrangian 3-star, not only if

$$
T_{x_{0}} S \cap T_{x_{0}} S_{1}=T_{x_{0}} S_{1} \cap T_{x_{0}} S_{2}=T_{x_{0}} S_{2} \cap T_{x_{0}} S_{3}=\{0\} .
$$

In general we write

$\tau\left(S, S_{1}, S_{2}\right)=n+\operatorname{dim}\left(T_{x_{0}} S \cap T_{x_{0}} S_{1}\right)+\operatorname{dim}\left(T_{x_{0}} S_{1} \cap T_{x_{0}} S_{2}\right)+\operatorname{dim}\left(T_{x_{0}} S_{2} \cap T_{x_{0}} S_{3}\right)(\bmod 2)$.

In the transversal case all 3 -stars are linearizable (cf. [6]).

An important class of Lagrangian submanifolds in $\mathcal{M}$, if $M_{1}=T^{\star} Q$ and $M_{2}=T^{\star} Q$, is formed by the cotangent bundle lifting $T^{\star} \Phi \subset \mathcal{M}$ of the smooth mappings $\Phi: Q \rightarrow Q$. In general we consider the symplectic relations $L_{(\Phi, F)}$ defined by the pair $(\Phi, F)$, where $\Phi: Q \rightarrow Q, F: Q \rightarrow \mathbb{R}$ are smooth mappings (cf. [8]). $L_{(\Phi, F)}$ is defined as a constrained Lagrangian submanifold in $\left(T^{\star}(Q \times Q), \pi_{2}^{*} \omega_{Q}-\pi_{1}^{*} \omega_{Q}\right)$ over graph $\Phi \subset Q \times Q$ with generating function $F$ extended into the whole $Q \times Q$. Now we have the following result.

Proposition 2.2. If $\sigma$ is a periodic orbit of $L_{(\Phi, F)}$ and of $T^{\star} \Phi$, then $F$ has a critical point at each point $x_{i} \in \sigma$ and $N(\sigma)$ is the sum of the corresponding critical values:

$$
N(\sigma)=\sum_{i=0}^{k-1} F\left(x_{i}\right) .
$$

P r o of. We see that for any periodic orbit $\sigma$ of the simple lifting $T^{\star} \Phi$ the symplectic invariant $N(\sigma)=0$. So if we demand the same orbit to be periodic for $L_{(\Phi, F)}$ (say affine lifting of $\Phi)$, then the function $F$ should have the critical points at every point of the orbit. In fact we check it locally writing the generating family for $L_{(\Phi, F)}$, namely

$$
G(x, y, \lambda)=\sum_{i=1}^{n}\left(y_{i}-\phi_{i}(x)\right) \lambda_{i}+F(x) \text {. }
$$


ExAmple 2.1. We may take $Q=\mathbb{R}^{\nvdash}$ and the map $\Phi\left(x_{1}, x_{2}\right)=\left(-x_{1}, x_{2}^{2}\right)$, so

$T^{\star} \Phi=\left\{\left(\left(x_{1}, x_{2}, p_{1}, p_{2}\right),\left(y_{1}, y_{2}, \xi_{1}, \xi_{2}\right)\right): y_{1}=-x_{1}, y_{2}=x_{2}^{2}, p_{1}=-\xi_{1}, p_{2}=2 \xi_{2} x_{2}\right\}$.

We consider all two-point orbits $\sigma$ of the type

$$
\left(\left(x_{10}, 0, p_{10}, 0\right),\left(-x_{10}, 0,-p_{10}, 0\right)\right) .
$$

Taking the generating function $F\left(x_{1}, x_{2}\right)=\cos x_{1}+\cos x_{2}$ we find

$$
N(\sigma)=(-1)^{k} 2
$$

for

$$
\sigma=\left\{\left(\left(k \pi, 0, p_{10}, 0\right),\left(-k \pi, 0,-p_{10}, 0\right)\right)\right\} .
$$

If $x_{0} \in M$, then periodic orbits of $L$ from the point $x_{0} \in L(M) \cap L^{t}(M)$ are determined by the strata $L_{i}\left(x_{0}\right), i \in N$ of the space

$$
L\left(x_{0}\right)=\left\{x \in M:\left(x_{0}, x\right) \in L\right\} .
$$

We define

$$
\begin{aligned}
L_{1}\left(x_{0}\right) & =L\left(x_{0}\right) \cap\left\{x_{0}\right\}, \\
L_{2}\left(x_{0}\right) & =L\left(x_{0}\right) \cap L^{t}\left(x_{0}\right), \\
\ldots & \cdots \cdots \\
L_{k}\left(x_{0}\right) & =L\left(x_{0}\right) \cap\left(L^{t}\right)^{k-1}\left(x_{0}\right) .
\end{aligned}
$$

The set of those points of $L\left(x_{0}\right)$ which do not belong to any $L_{k}\left(x_{0}\right)$ for some finite $k$ is denoted by $L_{\infty}\left(x_{0}\right)$. The set of exactly $k$-point periodic orbits from $x_{0}$ is written in the following way:

$$
\widetilde{L}_{2}\left(x_{0}\right)=\left\{x \in L\left(x_{0}\right):\left(x, x_{0}\right) \in L\right\}
$$

and

$$
\begin{aligned}
& \widetilde{L}_{k}\left(x_{0}\right)=\left\{x \in L\left(x_{0}\right)-\bigcup_{i=2}^{k-1} \widetilde{L}_{i}\left(x_{0}\right):\right. \\
& \left.\exists\left(x_{1}, x_{2}, \ldots, x_{k-2}\right) \in\left(L(x), L\left(x_{1}\right), \ldots, L\left(x_{k-3}\right)\right) \text { such that }\left(x_{k-2}, x_{0}\right) \in L\right\} \text { for } k>2 .
\end{aligned}
$$

3. The Lagrangian Grassmannian. Now we consider the linear product symplectic space

$$
\mathcal{M}=\left(M \times M, \pi_{2}^{*} \omega-\pi_{1}^{*} \omega\right),
$$

where $(M, \omega)$ is a $2 n$-dimensional symplectic vector space. By $\Lambda_{2 n}$ we denote the Lagrangian Grassmannian of linear subspaces in $\mathcal{M}$, and by $M_{1}$ and $M_{2}$ - the symplectic spaces canonically placed in $\mathcal{M}, M_{1}=M \times\{0\}, M_{2}=\{0\} \times M$. Equivalently we write

$$
\left(M_{1} \times M_{2}, \pi_{2}^{*} \omega_{2}-\pi_{1}^{*} \omega_{1}\right)
$$

for $\mathcal{M}$, where

$$
\omega_{2}=\pi_{2}^{*} \omega-\left.\pi_{1}^{*} \omega\right|_{\{0\} \times M}, \quad-\omega_{1}=\pi_{2}^{*} \omega-\left.\pi_{1}^{*} \omega\right|_{M \times\{0\}} .
$$

At first we have the natural decomposition. 
Proposition 3.1. If $L \in \Lambda_{2 n}$ then we have the two excluding each other possibilities: either $L$ is transversal to $M_{1}$ and also $L$ is transversal to $M_{2}$ or $L$ is not transversal to $M_{1}$ and also $L$ is not transversal to $M_{2}$.

Proof. If $L$ is transversal to $M_{2}$ then it may be parametrized by $M_{1}$ so $L$ is a graph of a maximal rank symplectic mapping $M_{1} \rightarrow M_{2}$ and so has to be transversal to $M_{1}$ (one can replace $M_{2}$ by $M_{1}$ in this argument). If $L$ is not transversal to $M_{1}$, then assuming that $L$ is transversal to $M_{2}$ on the basis of previous argument we get the transversality of $L$ to $M_{1}$, which contradicts our assumption.

The critical subset of $\Lambda_{2 n}$ is defined as the set $C \Lambda_{2 n}$ of those Lagrangian subspaces which are not transversal to both subspaces $M_{1}$ and $M_{2}$.

$C \Lambda_{2 n}=\left\{L \in \Lambda_{2 n}: L\right.$ is not transversal to $M_{1}$ and also $L$ is not transversal to $\left.M_{2}\right\}$.

Elements of $C \Lambda_{2 n}$ cannot be obtained as the graphs of linear symplectic transformations between $M_{1}$ and $M_{2}$.

The supercritical set of $\Lambda_{2 n}$ is defined as the Cartesian product

$$
S \Lambda_{2 n}=\Lambda_{n} \times \Lambda_{n} \subset \Lambda_{2 n} .
$$

These are Lagrangian subspaces $L=\left(W_{1}, W_{2}\right)$ where $W_{1}$ and $W_{2}$ are Lagrangian subspaces in $\left(M_{1}, \omega_{1}\right)$ and $\left(M_{2}, \omega_{2}\right)$ respectively.

We find

$$
\operatorname{codim} S \Lambda_{2 n}=n^{2} .
$$

Proposition 3.2. If $L \in C \Lambda_{2 n}$ then $L$ has a decomposition

$$
L=R_{2}^{t} \circ \widetilde{L} \circ R_{1}
$$

where $\widetilde{L}, R_{1}, R_{2}$ are linear Lagrangian subspaces

$\widetilde{L} \subset\left(\widetilde{M}_{1} \times \widetilde{M}_{2}, \pi_{2}^{*} \widetilde{\omega}_{2}-\pi_{1}^{*} \widetilde{\omega}_{1}\right), R_{1} \subset\left(M_{1} \times \widetilde{M}_{1}, \pi_{2}^{*} \widetilde{\omega}_{1}-\pi_{1}^{*} \omega\right), R_{2} \subset\left(M_{2} \times \widetilde{M}_{2}, \pi_{2}^{*} \widetilde{\omega}_{2}-\pi_{1}^{*} \omega\right)$, $R_{1}, R_{2}$ are graphs of projections $\rho_{1}$ and $\rho_{2}$ onto $\widetilde{M}_{1}$ and $\widetilde{M}_{2}$, respectively,

$$
\rho_{1}^{*} \widetilde{\omega}_{1}=\left.\omega\right|_{\pi_{1}(L)}, \quad \rho_{2}^{*} \widetilde{\omega}_{2}=\left.\omega\right|_{\pi_{2}(L)},
$$

and $\widetilde{L} \in \Lambda_{2 n-2 k}-C \Lambda_{2 n-2 k}$ for some $k \in N$.

Proof. If $L \in C \Lambda_{2 n}$ then $\pi_{1}(L) \subseteq V_{1}, \pi_{2}(L) \subseteq V_{2}$, where $V_{1}, V_{2}$ are hypersurfaces in $M_{1}$ and $M_{2}$ respectively. If there is an equality then $V_{1}$ and $V_{2}$ are coisotropic so we have the natural projections $\rho_{i}$ along the symplectic polars $V_{1}^{L} \subset V_{1}, V_{2}^{L} \subset V_{2}$ onto the symplectic reduced spaces $\widetilde{M}_{1}=\left(V_{1} / V_{1}^{L}, \widetilde{\omega}_{1}\right), \widetilde{M}_{2}=\left(V_{2} / V_{2}^{L}, \widetilde{\omega}_{2}\right)$. So we represent $L$ uniquely by two hyperspaces $V_{i}$ and the Lagrangian subspace $\widetilde{L} \in \Lambda_{2 n-2}$ in $\left(\widetilde{M}_{1} \times \widetilde{M}_{2}, \pi_{2}^{*} \widetilde{\omega}_{2}-\pi_{1}^{*} \widetilde{\omega}_{1}\right)$. If $\widetilde{L} \in C \Lambda_{2 n-2}$ then we may proceed in an analogous way and obtain the noncritical representation for $\widetilde{L}$.

EXAMPLE 3.1. If $n=2$ we have only two strata of the singular set $C \Lambda_{4}$ : The first maximal stratum $C_{1} \Lambda_{4}$ is determined by the pair of two coisotropic subspaces, $V_{1}$ in $M_{1}$ and $V_{2}$ in $M_{2}$ and the symplectic linear map between the corresponding reduced symplectic spaces, $\operatorname{dim} C_{1} \Lambda_{4}=9$. The second stratum is $S \Lambda_{4}, \operatorname{dim} S \Lambda_{4}=6$.

In general we have the following result on the structure of the singular set $C \Lambda_{2 n}$. 
Proposition 3.3. We have the following partition into the smooth submanifolds

$$
C \Lambda_{2 n}=\bigcup_{k=1}^{n} C_{k} \Lambda_{2 n}
$$

where the elements of $C_{k} \Lambda_{2 n}$ are determined by the pairs of two coisotropic subspaces $V_{1}$ in $M_{1}$ and $V_{2}$ in $M_{2}$ of codimension $k$ and the symplectic linear automorphism of the $(2 n-2 k)$-dimensional symplectic space. In this partition $C_{n} \Lambda_{2 n}=S \Lambda_{2 n}$.

Proof. In fact it follows from the property that the projection of $L \in \Lambda_{2 n}$ onto $M_{1}$ and $M_{2}$ is always coisotropic (or Lagrangian). Thus starting from the hypersurfaces we see that the corresponding $\widetilde{L} \in \Lambda_{2 n-2}$ in the product of reduced symplectic spaces projects onto them or onto hypersurfaces in the more degenerated case. Repeating this argument for further representations of $L$ we get the natural decomposition by coisotropic subspaces of equal dimensions and linear symplectic maps in a symplectic space of respectively smaller dimension.

Corollary 3.1 .

$$
\operatorname{codim} C_{k} \Lambda_{2 n}=k^{2}, \quad k=1, \ldots, n .
$$

Proof. We calculate the dimension of the isotropic Grassmannian $I_{k}^{2 n}$ of $k$-isotropic planes in $2 n$-dimensional symplectic space $V$ (cf. [12]).

$$
\operatorname{dim} I_{k}^{2 n}=2 n k-\frac{1}{2} k(3 k-1) .
$$

This is the dimension of the corresponding space of $(2 n-k)$-dimensional coisotropic subspaces in $V$. Since $\operatorname{dim} \Lambda_{2 n}=2 n^{2}+n$, we get

$$
\begin{aligned}
\operatorname{codim} C_{k} \Lambda_{2 n} & =\operatorname{dim} \Lambda_{2 n}-2 \operatorname{dim} I_{k}^{2 n}-\operatorname{dim}\left(\Lambda_{2 n-2 k}\right) \\
& =n(2 n+1)-2\left(2 n k-\frac{1}{2} k(3 k-1)\right)-(n-k)(2 n-2 k+1)=k^{2} .
\end{aligned}
$$

4. Local classification. Let $(L, p)$ be a germ of a symplectic relation (Lagrangian submanifold) in $\mathcal{M}$. Now we introduce the natural equivalence group acting on the space of such germs.

Definition 4.1. We say that two germs $\left(L_{1}, p_{1}\right),\left(L_{2}, p_{2}\right)$ of symplectic relations in $\mathcal{M}$ are equivalent if there exist two symplectomorphism germs $B_{1}:\left(M_{1}, \pi_{1}\left(p_{1}\right)\right) \rightarrow$ $\left(M_{1}, \pi_{1}\left(p_{2}\right)\right)$ and $B_{2}:\left(M_{2}, \pi_{2}\left(p_{1}\right)\right) \rightarrow\left(M_{2}, \pi_{2}\left(p_{2}\right)\right)$ such that the symplectomorphism $B_{1} \times B_{2}$ of $\mathcal{M}$ sends $L_{1}$ into $L_{2}$ and $p_{1}$ into $p_{2}$.

For the symplectic relation $L \subset \mathcal{M}$ we define the corresponding symplectic "Gauss" map

$$
G: L \ni p \mapsto T_{p} L \in \Lambda_{2 n}
$$

We call $L$ to be in general position (or generic) if $G$ is transversal to $C \Lambda_{2 n}=\bigcup_{k=1}^{n} C_{k} \Lambda_{2 n}$.

Definition 4.2. We say that $L$ has a $k$-vertical position at $p \in L$ if $G(p) \in C_{k} \Lambda_{2 n}$. We call $k$ a rank of $k$-vertical position. A 0 -vertical position corresponds to the case of the graph of local symplectomorphism for $L$ at $p$, i.e. $G(p) \in \Lambda_{2 n}-C \Lambda_{2 n}$. 
Now we have the following restriction for vertical points.

Proposition 4.1. For generic $L$ the isolated points of vertical position appear only if $n=2 s^{2}, s \in N$. In this case they are points in $2 s$-vertical position. In their neighbourhood there are $k$-vertical positioned points with $k \leq 2 s$.

The standard representation of Lagrangian germs yields the following preparatory lemma.

LEMMA 4.1. For any germ $(L, p) \subset \mathcal{M}$ there are local cotangent bundle structures around $\pi_{1}(p)$, say $T^{*} X_{1}$, and around $\pi_{2}(p)$, say $T^{*} X_{2}$, such that $(L, p)$ is generated in

$$
\mathcal{M} \cong\left(T^{*} X_{1} \times T^{*} X_{2}, \pi_{2}^{*} \omega_{X_{2}}-\pi_{1}^{*} \omega_{X_{1}}\right)
$$

by the germ of a generating function $F:\left(X_{1} \times X_{2}, \pi_{X_{1} \times X_{2}}(p)\right) \rightarrow \mathbb{R}$, such that, in local coordinates on $\left(X_{1} \times X_{2}, \pi_{X_{1} \times X_{2}}(p)\right)$, we have

$$
F(x, y)=\sum_{i, j=1}^{n} x_{i} y_{j} \phi_{i j}(x, y) .
$$

Proof. If $((p, q),(\tilde{p}, \tilde{q}))$ are Darboux coordinates on $\mathcal{M}$, then we find the partition $I \cup J=\{1, \ldots, n\}, I \cap J=\emptyset, \tilde{I} \cup \tilde{J}=\{1, \ldots, n\}, \tilde{I} \cap \tilde{J}=\emptyset$, such that there exists a smooth function $S\left(p_{I}, q_{J}, \tilde{p}_{\tilde{I}}, \tilde{q}_{\tilde{J}}\right)$, which generates $(L, p)$. By the symplectomorphism

$$
\Phi(p, q ; \tilde{p}, \tilde{q})=\left(-q_{I}, p_{J}, p_{I}, q_{J} ;-\tilde{q}_{\tilde{I}}, \tilde{p}_{\tilde{J}}, \tilde{p}_{\tilde{I}}, \tilde{q}_{\tilde{J}}\right)=(\xi, x ; \eta, y),
$$

which preserves the product structure of $\mathcal{M}$ we find the generating function $F(x, y)$ for $(L, p)$ in canonical special symplectic structure $T^{*} X_{1} \times T^{*} X_{2}$ on $\mathcal{M}$. Then using the symplectomorphisms of $M_{1}$ and $M_{2}$ preserving the corresponding cotangent bundle structures we obtain the reduced form (4) of function $F$.

Proposition 4.2. Let $p \in L$, then we have:

1. The rank of the vertical position of $L$ at $p$ is equal to the corank of the matrix $\left(\phi_{i j}=\frac{\partial^{2} F}{\partial x_{i} \partial y_{j}}\right)$ at $\pi_{X_{1} \times X_{2}}(p)$, it is the symplectic invariant of $(L, p) \subset \mathcal{M}$.

2. At each $p \in L$, for a generic $L$ the family of mappings

$$
\Phi: X_{1} \times X_{2} \rightarrow \mathbb{R}^{\ltimes}, \quad(\curvearrowleft, \curvearrowright)=\left(\sum_{\beth=\nVdash}^{\ltimes} \curvearrowright_{\beth} \phi_{\nVdash\rfloor}, \ldots, \sum_{J=\nVdash}^{\ltimes} \curvearrowright_{\beth} \phi_{\ltimes\rfloor}\right)
$$

has a generic singularity at $\pi_{X_{1} \times X_{2}}(p)$.

Proof.

1. Any linear relation $L$ is equivalent to one generated by the quadratic form $\sum_{i, j=1}^{n} x_{i} y_{j} a_{i j}$, so the dimension of its kernel is exactly equal to the rank of verticality of $L$. This is a local symplectic invariant of $(L, p)$, which does not depend on the choice of the corresponding cotangent bundle structures.

2. Any relation $L$ is locally represented by a smooth family of mappings $\Phi(x, y)=$ $\left(\widehat{\phi}_{1}(x, y), \ldots, \widehat{\phi}_{n}(x, y)\right)$, such that $\Phi(x, 0) \equiv 0$. We see that the Gauss map $G: L \rightarrow$ $T_{p} L$ corresponds exactly to the one-jet extension $j^{1} \Phi(x, y)$ of the mapping $\Phi$, so the transversality of $G$ is equivalent to the corresponding transversality of $\Phi$ to the canonical stratification of smooth mappings of $\mathbb{R}^{\ltimes} \times \mathbb{R}^{\ltimes}$ into $\mathbb{R}^{\ltimes}$. 
Corollary 4.1. At any point $p \in L$ of the 0 -vertical position of $L$, the symplectic relation $L$ is parallelizable, i.e. it is locally symplectically equivalent to its tangent space $T_{p} L$ with the generating function

$$
F(x, y)=\sum_{i=1}^{n} x_{i} y_{i}
$$

Remark 4.1. If $n=2$ then the supercritical points appear in generic $L$ as the isolated points, in fact codim $C_{2} \Lambda_{4}=4$, and $G$ is transversal to $S \Lambda_{4}=C_{2} \Lambda_{4}$. If $p \in L$ is a supercritical transversal point then on the basis of Lemma 4.1, on a neighbourhood of $p, L$ is generated locally by the generating function

$$
F(x, y)=\sum_{i, j=1}^{2} x_{i} y_{j} \phi_{i j}(x, y),
$$

where $\phi_{i j}(0,0)=0, i, j=1,2, p=0$, and the transversality condition is equivalent to

$$
\operatorname{rank} D \Phi(0)=4 \text {, }
$$

where $\Phi(x, y)=\left(\phi_{i j}(x, y)\right) \in M_{2 \times 2}$.

If we need to iterate a symplectic relation $L$ we have to use the symplectic equivalence group preserving the canonical product structure of $\mathcal{M}=\left(M \times M, \pi_{2}^{*} \omega-\pi_{1}^{*} \omega\right)$. We say that two germs $\left(L_{1}, p_{1}\right),\left(L_{2}, p_{2}\right) \subset \mathcal{M}$, where $\pi_{1}\left(p_{i}\right)=\pi_{2}\left(p_{i}\right)=\tilde{p}_{i}, i=1,2$, are $D$-equivalent (diagonal equivalence) if there exists a symplectomorphism germ $B:\left(M, \tilde{p}_{1}\right) \rightarrow\left(M, \tilde{p}_{2}\right)$ such that $(B \times B)\left(L_{1}\right)=L_{2}$. Using the notation of composition of symplectic relations we can write

$$
L_{2}=\widehat{B} \circ L_{1} \circ \widehat{B}^{t} .
$$

Now using the proof of Lemma 4.1 we obtain the following result.

Lemma 4.2. For any germ $(L, p) \subset \mathcal{M}$ there exists a local cotangent bundle structure $T^{*} X$ (D-equivalence) around $\pi_{1}(p)$ such that $(L, p)$ is generated in

$$
\mathcal{M} \cong\left(T^{*} X \times T^{*} X, \pi_{2}^{*} \omega_{X}-\pi_{1}^{*} \omega_{X}\right)
$$

by a Morse Family germ $F:\left(X \times X \times \mathbb{R}^{\top}, \nvdash\right) \rightarrow \mathbb{R}$ (we assumed $\pi_{1}(p)=0$ ),

$$
F(x, y, \lambda)=\sum_{i=1}^{n} x_{i} \phi_{i}(x, y, \lambda)
$$

such that $k \leq \operatorname{dim} X$. If the integer $k$ is minimal then it is an invariant of D-equivalence symplectic group action.

We see that the linear symplectic relations in $\mathcal{M}$ are classified by the classes of linear mappings

$$
\Phi=\left(\phi_{1}, \ldots, \phi_{n}\right): \mathbb{R}^{\ltimes} \times \mathbb{R}^{\ltimes} \times \mathbb{R}^{\urcorner} \rightarrow \mathbb{R}^{\ltimes}
$$

with the standard equivalence relation

$$
\Xi:(x, y, \lambda) \rightarrow(A(x), Y(y), \Lambda(x, y, \lambda))
$$


and the equivalent $\Phi^{\prime}$ is given by

$$
\Phi^{\prime}(x, y, \lambda)=A^{\mathrm{T}} \Phi(A(x), B(y), \Lambda(x, y, \lambda)) .
$$

Remark 4.2. Let us assume that $L \subset \mathcal{M}=\left(T^{*} X \times T^{*} X, \pi_{2}^{*} \omega_{X}-\pi_{1}^{*} \omega_{X}\right)$ is generated by the smooth generating function $F:(x, y) \rightarrow F(x, y)$. Let $\sigma$ be a periodic orbit of $L, \pi_{X \times X}(\sigma)=\left\{\left(x_{0}, x_{1}\right),\left(x_{1}, x_{2}\right), \ldots,\left(x_{k-1}, x_{0}\right)\right\}$. We see that $\left(x_{0}, x_{1}, \ldots, x_{k-1}\right)$ is a critical point of the function

$$
G\left(x_{0}, x_{1}, \ldots, x_{k-1}\right)=F\left(x_{0}, x_{1}\right)+F\left(x_{1}, x_{2}\right)+\ldots+F\left(x_{k-1}, x_{0}\right),
$$

and the invariant $N(\sigma)$ is its critical value.

As an example we can consider the billiard mapping generating function (see Introduction)

$$
\widetilde{H}(x, y)=|y-x| \text {. }
$$

Let $\left\{x_{0}, x_{1}, \ldots, x_{k-1}\right\}$ be an element of $\underbrace{X \times \ldots \times X}_{k}-\{\Delta\}$, where $\Delta$ is a total diagonal in $\underbrace{X \times \ldots \times X}_{k}$ and $X=\partial \Omega$. Then the function

$$
G\left(x_{0}, x_{1}, \ldots, x_{k-1}\right)=\left|x_{1}-x_{0}\right|+\left|x_{2}-x_{1}\right|+\ldots+\left|x_{0}-x_{k-1}\right|
$$

has a critical point exactly in the periodic orbit of the symplectic billiard map. For $\partial \Omega=S^{1}$ these are regular polygons.

By $\{N(\sigma)\}^{k}$ we denote the set of symplectic invariants for $k$-point orbits $\sigma$. For the general billiard system, $L$ may not be the graph of any symplectomorphism. Let $\phi: X \rightarrow \mathbb{R}^{\ltimes}$ be an imbedding of a closed orientable surface and we assume that it is generic, i.e. the function

$$
\widetilde{H}_{\phi}(x, y)=|\phi(x)-\phi(y)|
$$

defined on $X \times X$ outside of the diagonal $\Delta$ has only nondegenerate critical points on $X \times X-\Delta$. We easily see that the critical points of $\widetilde{H}_{\phi}$ are, in fact, the 2-point orbits (double normals) of the possibly nonconvex billiard system. By the Morse inequalities (cf. $[13,16])$ we obtain the following estimation for the surface case.

Proposition 4.3. If $\phi$ is a generic imbedding of a surface of genus $g$, then we have the following lower bound for the number of 2-point invariants

$$
\#\{N(\sigma)\}^{2} \geq 2 g^{2}+3 g+3 .
$$

In the case of ellipsoid with three unequal axes $\#\{N(\sigma)\}^{2}=3$. If this is an imbedding of the torus we have at least eight 2-point invariants of the torus-like two-dimensional billiard system. In the three-dimensional billiard systems the lower bound for $\#\{N(\sigma)\}^{2}$ is expressed by the first Betti numbers of $X$. We leave further estimations of the length spectrum of $X$ and investigations of periodic orbits of the general symplectic relations to the forthcoming paper. 
Acknowledgments. I would like to thank Donal O'Shea and Vladimir Zakalyukin for helpful conversations. I also thank Jan Kowalski for his help in preparing the final version of the paper.

\section{References}

[1] V. I. Arnol'd, S. M. Guseřn-Zade, A. N. Varchenko, Singularities of Differentiable Maps I, Monogr. Math. 82, Birkhäuser, Boston, 1985.

[2] A. P. Fordy, A. B. Shabat, and A. P. Veselov, Factorization and Poisson correspondences, Teoret. Mat. Fiz. 105:2 (1995), 225-245; reprinted in: Theoret. and Math. Phys. 105 (1995), 1369-1386.

[3] V. Guillemin, R. B. Melrose, A cohomological invariant of discrete dynamical systems, in: E. B. Christoffel. The influence of his work on mathematics and the physical sciences, P. L. Butzer and F. Fehér (eds.), Birkhäuser, Basel, 1981, 672-679.

[4] V. Guillemin, S. Sternberg, Some problems in integral geometry and some related problems in micro-local analysis, Amer. J. Math. 101 (1979), 915-955.

[5] V. Guillemin, S. Sternberg, Symplectic Techniques in Physics, Cambridge Univ. Press, Cambridge, 1984.

[6] S. Janeczko, Classification of lagrangian stars and their symplectic invariants, J. Phys. A 31 (1998), 3677-3685.

[7] S. Janeczko, Generating families for images of Lagrangian submanifolds and open swallowtails, Math. Proc. Cambridge Philos. Soc. 100 (1986), 91-107.

[8] S. Janeczko, Constrained Lagrangian submanifolds over singular constraining varieties and discriminant varieties, Ann. Inst. H. Poincaré Phys. Théor. 46 (1987), 1-26.

[9] J. B. Keller, Rays, waves and asymptotics, Bull. Amer. Math. Soc. 84 (1978), 727-750.

[10] J. Martinet, Singularities of Smooth Functions and Maps, London Math. Soc. Lecture Note Ser. 58, Cambridge Univ. Press, Cambridge, 1982.

[11] S. Marvizi, R. Melrose, Spectral invariants of convex planar regions, J. Differential Geom. 17 (1982), 475-502.

[12] M. Mikosz, On classification of the linear Lagrangian and isotropic subspaces, Demonstratio Math. 30 (1997), 437-450.

[13] J. Milnor, Morse Theory, Ann. of Math. Stud. 51, Princeton Univ. Press, Princeton, 1963.

[14] A. S. Mishchenko, V. E. Shatalov, B. Yu. Sternin, Lagrangian manifolds and the method of the canonical operator (in Russian), Nauka, Moscow, 1978.

[15] R. Ranga Rao, On some explicit formulas in the theory of Weil representation, Pacific J. Math. 157 (1993), 335-371.

[16] F. Takens, J. White, Morse theory of double normals of immersions, Indiana Univ. Math. J. 21 (1971), 11-17.

[17] W. M. Tulczyjew, The Legendre transformation, Ann. Inst. H. Poincaré Sect. A 27 (1977), 101-114.

[18] A. Weinstein, Symplectic geometry, Bull. Amer. Math. Soc. (N.S.) 5 (1981), 1-13.

[19] A. Weinstein, Lectures on Symplectic Manifolds, CBMS Regional Conf. Ser. in Math. 29, Amer. Math. Soc., Providence, 1977; corrected reprint, 1979.

[20] V. M. Zakalyukin, R. M. Roberts, Stability of Lagrangian manifolds with singularities (in Russian), Funktsional. Anal. i Prilozhen. 26 (1992), no. 3, 28-34; English transl.: Funct. Anal. Appl. 26 (1992), 174-178. 